\title{
ANÁlisis FITOLÍTICOS DE LAS PRINCIPALES ESPECIES DE PALMERAS (ARECACEAE) PREsentes EN REgIONES SubTropicales de AMÉrica DEL SUR
}

\author{
NOELIA ISABEL PATTERER ${ }^{1,2^{*}}$
}

\begin{abstract}
Summary: Phytolith analyses of the main palm species (Arecaceae) present in subtropical regions of South America. The South American continent has become one of the largest centers of richness and diversity of the Arecaceae family. The palms are characterized by the presence of opal phytoliths, called stegmatas, with particular characteristics that identify many of the members of this family. The aim of this paper is to describe the phytolith assemblages of the palm species and search the diagnostic power through a biometric analysis performed to globular phytoliths. The results stated that the analysis of leaf phytolith assemblages shows variations that distinguish the different subfamilies selected. This raises its importance in the interpretation of fossil phytolith assemblages and palaeobotanical recognition of the members of this family by palaeoenvironmental study of sedimentary sequences.
\end{abstract}

Key Words: Biometric analysis, Phytoliths, Stegmatas.

Resumen: El continente sudamericano aparece como uno de los mayores centros de riqueza y diversidad de la familia Arecaceae. Las palmeras se caracterizan por la presencia de fitolitos de naturaleza silícea denominados stegmatas, los mismos presentan características particulares que permiten identificar a gran parte de los miembros de esta familia. El objetivo del presente trabajo es, describir las asociaciones fitolíticas de especies de palmeras y buscar el valor diagnóstico de las mismas a través de un análisis biométrico, a través de los fitolitos globulares. Los resultados expresaron que el análisis de las asociaciones fitolíticas foliares mostró variaciones que permitieron distinguir las distintas subfamilias seleccionadas. Esto plantea su importancia en la interpretación de asociaciones fitolíticas fósiles y el reconocimiento paleobotánico de los integrantes de esta familia, a través del estudio de secuencias sedimentarias.

Palabras clave: Análisis biométricos, Fitolitos, Estegmatas.

\section{INTRODUCCIÓN}

Entre los vegetales productores de fitolitos y en particular de silicofitolitos, las gramíneas y las ciperáceas son las familias de mayor importancia en lo que respecta a las características cualicuantitativas de producción (Piperno, 1988; Pearsall, 2000). No obstante ello, existen grupos vegetales cuya producción fitolítica puede resultar de importancia ya sea por una elevada cantidad de

${ }^{1}$ Laboratorio de Paleobotánica CICYTTP-CONICET, Dr. Materi y España s/n, Diamante (E3105BWA), Entre Ríos, Argentina.

2 Facultad de Ciencia y Tecnología, Universidad Autónoma de Entre Ríos, Argentina. ${ }^{*}$ E-mail: noepatt1@hotmail. com elementos con escasa variabilidad en sus formas o bien por el elevado valor diagnóstico de sus fitolitos. Para el caso de las palmeras, este grupo se caracteriza por la presencia de fitolitos de naturaleza silícea que en particular han sido denominados como stegmatas (Tomlinson, 1961). Según este autor los tipos morfológicos más frecuentes son los esféricos a elipsoidales, lisos, rugosos o espinosos, los fitolitos cónicos de superficie rugosa y las arenas de sílice. En general estas biomineralizaciones se originan en el lumen celular de células parenquimáticas del mesófilo foliar y tallo, y no copian la forma de la célula de caja en donde se originaron. Los dos tipos de cuerpos de sílice de mayor tamaño (los globulares y los cónicos), se disponen en filas adyacentes a los haces vasculares de los tallos y las hojas, en células particulares del mesófilo y de la hipodermis. Esto 
es en parte, lo que en palmeras origina la gran variabilidad de estas formas fitolíticas, a diferencia de lo que ocurre en otros grupos, como por ejemplo en las gramíneas, donde los fitolitos copian la forma de las células de caja en donde se originan.

Desde el punto de vista paleobotánico, las palmeras se encuentran representadas en el registro paleontológico por megafósiles (petrificaciones de tallos y frutos e impresiones foliares) y por microfósiles (granos de polen y fitolitos). Recientemente, la incorporación de análisis fitolíticos en sedimentos cenozoicos argentinos (Mazzoni, 1979; Zucol \& Brea, 2000 a, b; Zucol et al., 1999, 2001; Patterer, 2012), plantearon la necesidad de reconocer el valor diagnóstico de los morfotipos fitolíticos de las palmeras y su importancia en las interpretaciones paleobotánicas y paleoclimáticas. Es con esta finalidad, así como la de conocer los patrones de producción en los distintos órganos de estas plantas, que en la presente contribución se han implementado análisis morfométricos de los fitolitos de un grupo de palmeras, conjuntamente con el estudio de las distintas formas y cantidad de material biomineralizado en los distintos órganos. Análisis similares en especies de otros grupos de Arecaceae han permitido la diferenciación de los fitolitos producidos por palmeras (Albert et al., 2009). Los distintos estudios paleobotánicos coinciden en considerar a las palmeras como un grupo de importancia paleoclimática (Manchester et al., 2010; Martínez, 2010; Zucol et al., 2010), dadas sus características fitogeográficas y requerimientos ecológicos y climáticos.

Con la finalidad de profundizar en el conocimiento de la producción fitolítica de este grupo, se estudió la producción fitolítica y se realizó el análisis biométrico de los fitolitos globulares presentes en los distintos órganos de Syagrus romanzzoffiana (hoja, pecíolo, espata, inflorescencia y fruto). Éste estudio preliminar, se realizó para conocer la variabilidad de tamaños de los fitolitos globulares en cada órgano. Posteriormente se llevo a cabo un análisis comparativo de las asociaciones fitolíticas de las principales especies de Arecaceae cuyas distribuciones llegan a áreas subtropicales de América del Sur. El objetivo del presente trabajo es el de buscar el poder diagnóstico de los fitolitos globulares, con el propósito de ser aplicado principalmente a secuencias sedimentarias, en estudios paleoambientales.

\section{Materiales y Métodos}

\section{Selección de la muestra}

Para la selección de las especie se ha tenido como premisa, ubicar las especies que logran llegar en su distribución a regiones subtropicales de América del Sur (Fig.1). Del arco subtropical que abarca Argentina, Uruguay y Chile, y de acuerdo a la descripción de los integrantes sudamericanos de esta familia (Uhl \& Dransfield, 1987; Uhl et al., 1995) las especies presentes en esta área pertenecen a las subfamilias Coryphoideae y Arecoideae. Las Coryphoideae están representadas por los géneros Copernicia y Trithrinax (tribu Corypheae); mientras que las Arecoideae por Euterpe (tribu Areceae, subtribu Euterpeinae), Butia, Jubaea, Syagrus y Allagoptera (tribu Cocoeae, subtribu Butiinae) y Acrocomia (tribu Bactridinae).

Se seleccionaron 11 especies: Trithrinax schizophylla Drude., Trithrinax campestris (Burmeister) Drude y Griseb. ex Griseb., Trithrinax brasilensis Mart., Copernicia alba Morong., Euterpe edulis Mart., Butia capitata (Mart.) Becc., Butia yatay (Mart.) Becc., Syagrus romanzoffiana (Cham.) Glassman.; Allagoptera campestris (Mart.) Kuntze Acrocomia aculeata (Jacq.) Lodd. ex Mart y Jubeae chilensis (Molina) Baill. El material utilizado proviene de recolecciones personales, así como de ejemplares del Herbario del Museo Argentino de Ciencia Naturales "Bernardino Rivadavia" (BA).

Se muestrearon entre ocho y diez ejemplares por especie, sobre los cuales se realizaron los muestreos. En primer lugar se seleccionó una especie de amplia distribución y de porte adecuado para un muestreo múltiple: Syagrus romanzzoffiana. Los mismos fueron examinados con la finalidad de establecer si existían variaciones composicionales en las asociaciones fitolíticas de los distintos órganos de la planta, para ello se obtuvieron muestras de hoja, pecíolo, espata, inflorescencia y fruto, en cada uno de los distintos ejemplares.

\section{Extracción de fitolitos}

La obtención de los fitolitos se realizó mediante la técnica de calcinación de Labouriau (1983), la cual consiste en un lavado previo del material con detergente neutro, a los fines de remover desechos extraños y facilitar el ablandamiento del material herborizado. Estas muestras previamente pesadas, se carbonizan en mufla a $200^{\circ} \mathrm{C}$ durante 2 horas; 

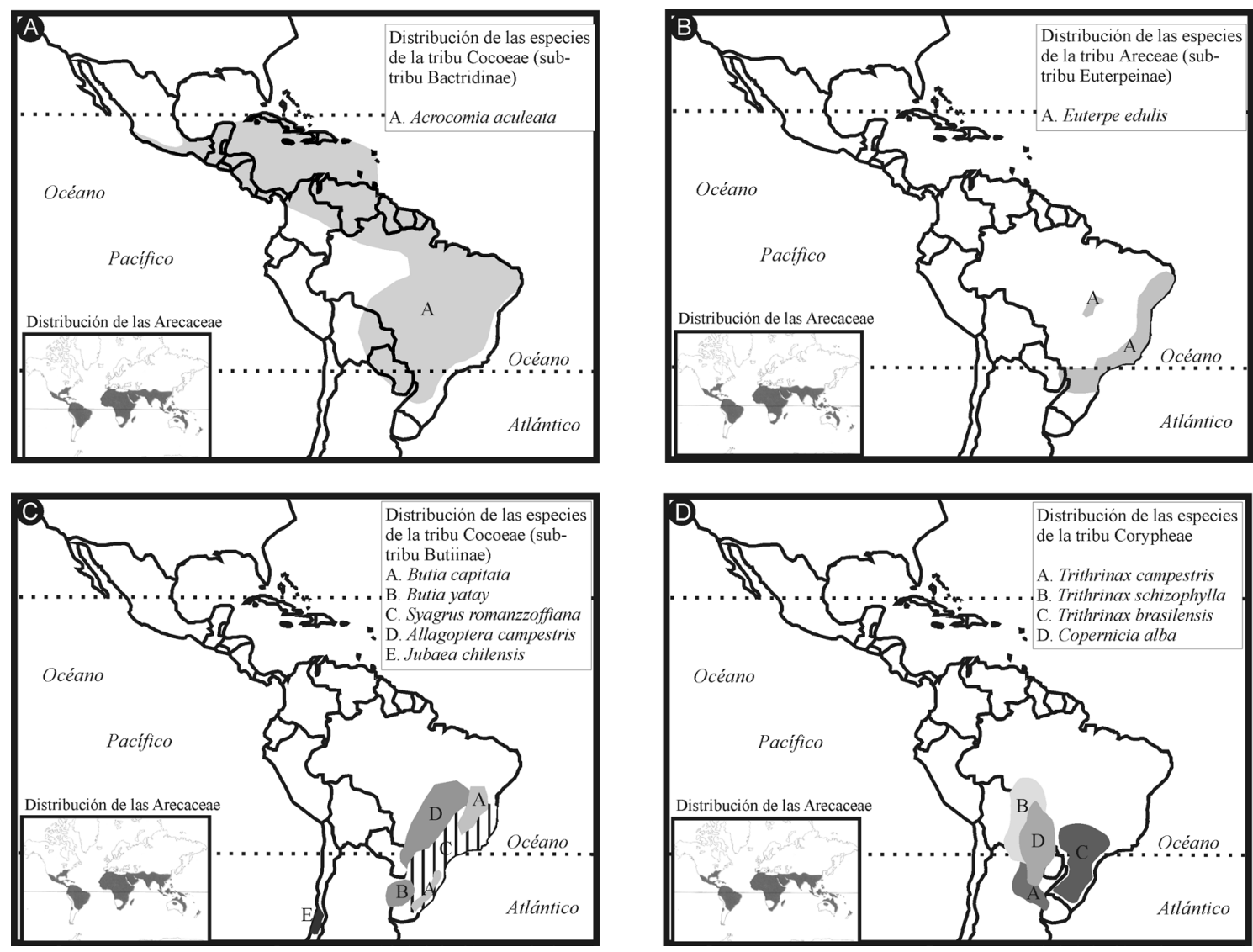

Fig. 1. Mapas de distribución de las especies seleccionadas para el análisis fitolítico (Modificado de Henderson et al., 1995).

posteriormente se trituró el material y se le realizó una digestión química con $100 \mathrm{ml}$ de una solución $5 \mathrm{~N}$ de HCL, durante 10-30 minutos a fuego directo hasta ebullición, se filtró el material resultante mediante filtro de papel libre de cenizas y se calcinó a $800^{\circ} \mathrm{C}$ durante 2 horas. Las cenizas obtenidas fueron pesadas antes de realizar los preparados microscópicos utilizando Bálsamo de Canadá como medio de montaje, el porcentaje de las mismas para cada muestra se obtuvo considerando la diferencia entre el peso de ceniza obtenido y el peso de materia seca del vegetal muestreado. Las cenizas fueron depositadas en el repositorio de cenizas del laboratorio de Paleobotánica, CICYTTP, Diamante, Entre Ríos.

\section{Medición y recuento}

La medición y recuento de los elementos se realizó en un microscopio óptico Nikon Labophot, mientras que para una observación de detalle se utilizó un microscopio electrónico de barrido Phillips XL30, del servicio de Microscopía del Museo Argentino de Ciencias Naturales Bernardino Rivadavia.

Con la finalidad de establecer el poder diagnóstico de las palmeras a través de sus asociaciones fitolíticas, se establecieron las variaciones biométricas de los fitolitos globulares, de esta forma se midió el diámetro mayor (diámetro que incluye las espinas que cubren la superficie sensu Albert et al., 2009; Patterer et al., 2009), de los elementos globulares característicos de la familia Arecaceae. Por cada especie se contaron y midieron entre 300 y 400 fitolitos globulares, con la finalidad de encontrar diferencias ya sea entre los órganos de un mismo ejemplar o entre las muestras foliares de las diferentes especies. Los datos fueron 
procesados por medio de estadísticos descriptivos y multivariados, utilizando el programa PAST, PAleontological STatistics (Hammer et al. 2001).

\section{Resultados}

Análisis fitolíticos en órganos de Syagrus romanzzoffiana

El análisis del contenido fitolítico (gr de cenizas por gr de materia seca) por órgano de los ejemplares de $\boldsymbol{S}$. romanzzoffiana, mostró que las pinnas presentan la mayor cantidad de sílice $(0,5-0,6$ gr), mientras que los pecíolos (0,3-0,4 gr) y las vainas $(0,2-0,3 \mathrm{gr})$ poseen una relación de biomineralización relativamente menor; por último, los valores más bajos se han observado en espata, inflorescencia y fruto (0,05-0,15 gr) (Fig. 2-A). En lo referente a los fitolitos, las muestras de hoja, pecíolo, espata, inflorescencia y fruto presentaron
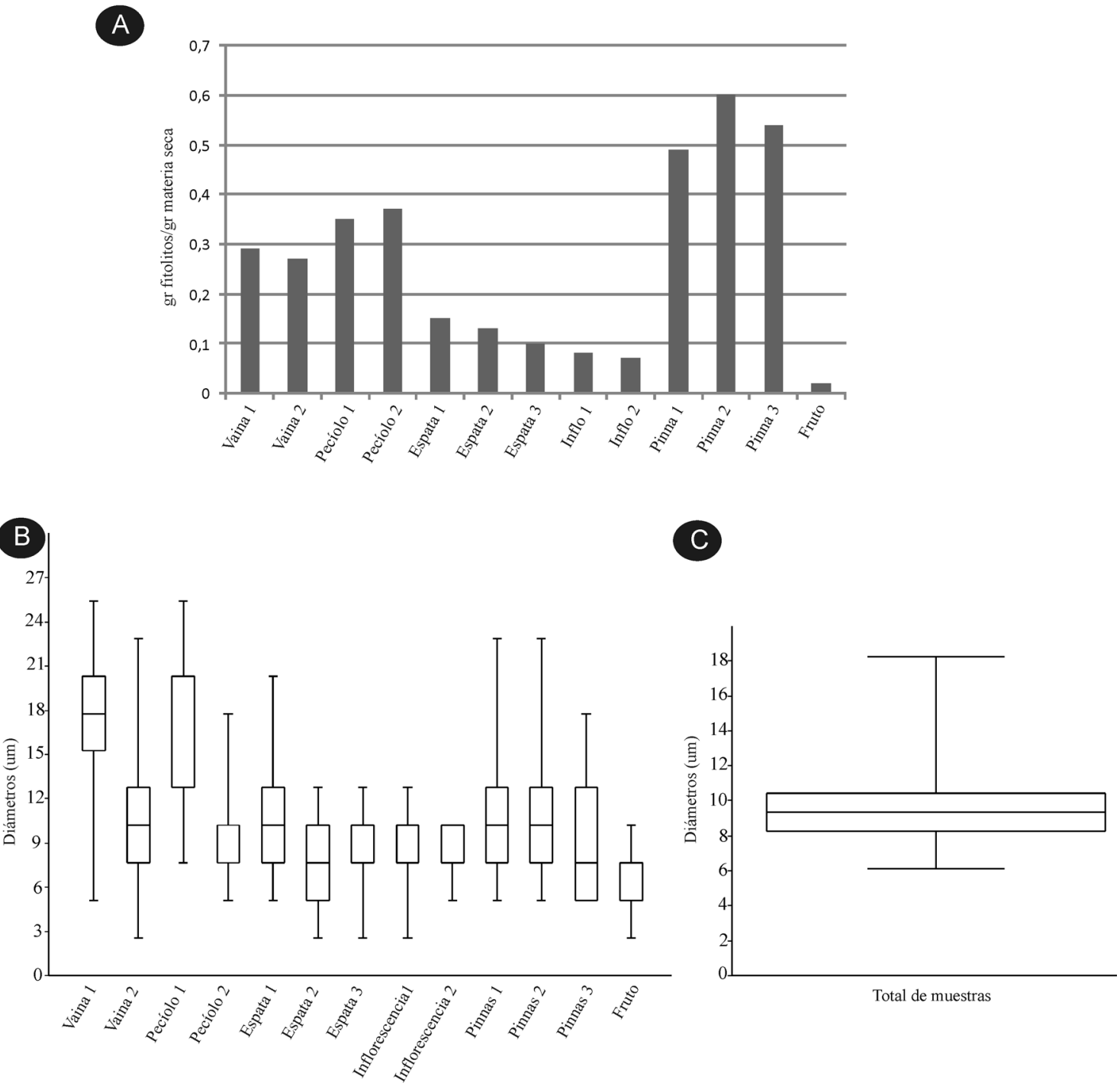

Fig. 2. A: Gráficos de barras mostrando gr de ceniza por gr de materia seca. B: Gráficos de caja obtenidos a partir de la media del diámetro de los fitolitos globulares de pinna, espata, vaina, pecíolo, fruto e inflorescencia de S. romanzzoffiana. C: Gráfico de caja obtenido mediante la media ponderada a partir del total de las muestras. 
silicofitolitos de manera abundante, principalmente de formas globulares, aisladas o articuladas, con tamaños que oscilan entre los 2 a los $25 \mu \mathrm{m}$ de diámetro. También se observaron en menor abundancia, elementos elipsoidales espinosos así como elementos de conducción silicificados y arenas silíceas (cuerpos pequeños de sílice probablemente pertenecientes a las estegmatas, que fueron separados de la misma cuando se seccionó la lámina foliar) de manera dispersa y/o aglomerada.

Con respecto a los diámetros de los fitolitos globulares, los valores de rango como la media muestral (Tabla 1), permiten observar variaciones diferenciales entre las sub-muestras tomadas por cada órgano (Fig. 2-A) con valores medios que oscilan entre los 7,30-18,25 $\mu \mathrm{m}$. Las pinnas por su parte, han presentado el mayor grado de homogeneidad, tanto por sus medias como por sus rangos. La media ponderada obtenida mediante estos valores (9,8 $\mu \mathrm{m}$ (Fig. 2-C) permite observar que los valores estadísticos para esta especie se ajustan principalmente a los obtenidos en los fitolitos globulares de las pinnas, los cuales se encuentran también presentes en algunas muestras de vaina, espata e inflorescencia.

Del análisis realizado en distintos órganos de $S$. romanzzoffiana, se observó que la hoja, es la parte de la planta con mayor cantidad de biomineralización (Fig. 2-A), por lo tanto es la que mayor cantidad de restos silíceos contiene, sumado a ello, el tamaño de los fitolitos globulares poseen una media acorde con la media ponderada obtenida para este morfotipo fitolítico en el resto de la planta, por lo cual puede ser considerado representativo del existente en todo el vegetal (Fig. 2-B).

\section{Análisis de las especies de palmeras}

De la comparación de la relación de la cantidad de biomineralizaciones por gramo de materia seca entre las especies estudiadas, $B$. yatay presentó la mayor relación $(0,25 \mathrm{gr})$, mientras que $S$. romanzzoffiana y $T$. campestris $(0,15 \mathrm{gr})$ con relaciones menores son las especies con mayores porcentajes de biomineralizaciones, en menor abundancia lo realizan C. alba (0,09 gr), A. aculeata $(0,08$ gr) y E. edulis $(0,07 \mathrm{gr})$, siendo J. chilensis $(0,04 \mathrm{gr})$ la especie que menor cantidad de biomineralizaciones en ceniza produce.

Desde el punto de vista anatómico, entre los géneros analizados de la subfamilia Coryphiodeae, Trithrinax presenta fitolitos muy abundantes en la base foliar y escasos en la lámina foliar, se disponen en hileras cortas, discontinuas, adyacentes a las venas (Fig. $3 \mathrm{~N}$ y M; Fig. $5 \mathrm{~B}$ y C); mientras que en Copernicia los fitolitos se disponen en largas hileras discontinuas comúnmente adyacentes a los haces vasculares foliares (Tomlinson, 1961) (Fig. 4 C-F; Fig. 5 D y E).

En la subfamilia Arecoideae, subtribu Butiinae, los fitolitos se caracterizan por encontrarse en la hoja de manera abundante, dispuestos en hileras discontinuas como es el caso de Butia (Fig. 3A-G; Fig. 5A) o en hileras más o menos continuas adyacentes a las venas, asociados a fibras longitudinales adaxiales, en Syagrus (Fig. 3H-L; Fig.5 F y G). En ambos casos los fitolitos son de morfología esférica a elipsoidal con su superficie cubierta de cortas espinas. Acrocomia (subtribu Bactridinae) se caracteriza por presentar fitolitos de forma cónica, de base irregular o aplanada (Fig. 4G-I; Fig. 5J). Finalmente, dentro de la subtribu Euterpeinae, Euterpe se identifica por

\begin{tabular}{|lccccccccccccc|c|}
\multicolumn{10}{c}{ Tabla 1. Estadísticos descriptivos de las diferentes muestras correspondientes a Syagrus } \\
romanzzoffiana.
\end{tabular}




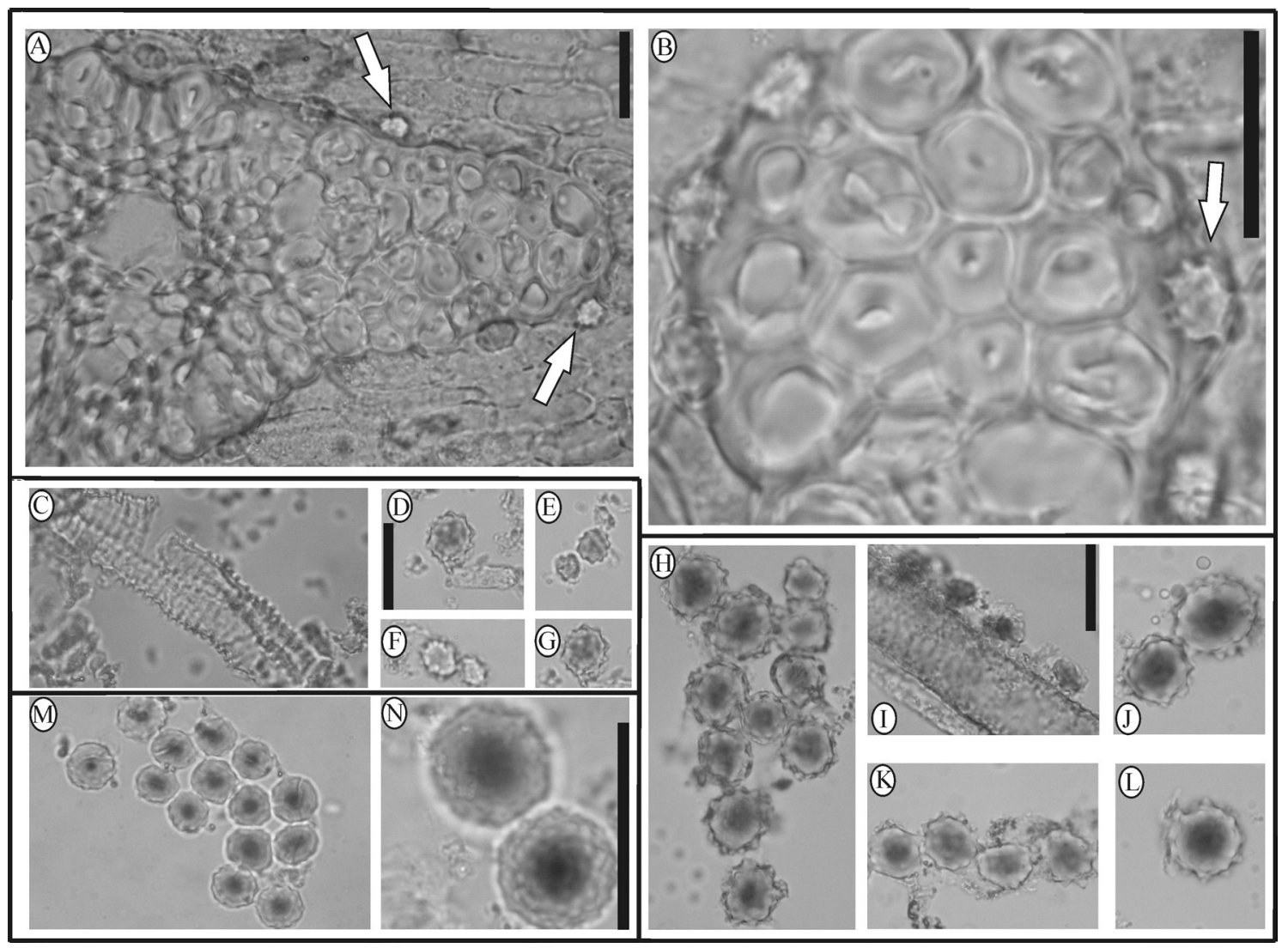

Fig. 3. A-B: Vista de las stegmatas adyacentes a los haces vasculares (Corte transversal de hoja de Butia yatay). C: elementos de conducción. D-G: elementos globulares espinosos (Cenizas de hoja de Butia yatay); H, J, K: Elementos globulares articulados. I: Elementos globulares asociados a elementos de conducción. L: Elemento globular espinoso aislado (Cenizas de hoja de Syagrus romanzzoffiana). M-N: Elementos globulares articulados de superficie levemente espinosa (Cenizas de hoja de Trithrinax campestris). Escala gráfica: A, B, D, I, N: $20 \mu \mathrm{m}$.

poseer fitolitos dispuestos en largas hileras continuas comúnmente adyacentes a los haces vasculares foliares (Tomlinson, 1961) (Fig. 4 A y B).

Con respecto a los fitolitos de las especies examinadas, en la mayoría de los casos, con excepción de $A$. aculeata, que solo presenta fitolitos cónicos, para el resto de las especies, se observó que definen la asociación de forma frecuente, fitolitos globulares, principalmente de superficie espinosa, acompañados en algunos casos por fitolitos elipsoidales y de forma escasa se observaron fitolitos originados a partir de elementos de conducción, así como complejos estomáticos, arenas silíceas, agrupadas o aisladas, las cuales fueron observadas en todas las especies en menor o mayor medida (Tabla 2).
Morfología de los elementos globulares en los diferentes géneros

El análisis estadístico de los diámetros de los fitolitos globulares espinosos (Fig. 6), permite observar una diferencia marcada con respecto a los valores medios obtenidos, presentando homogeneidad aquellos que oscilaron entre 2,5 a $22,9 \mu \mathrm{m}$ con variaciones en las distintas especies, siendo $S$. romanzzoffiana, la única especie que presentó fitolitos en ese rango total. Acrocomia, se diferencia del resto de las especies estudiadas, ya que es el único género que posee fitolitos cónicos, que resultan ser diagnósticos de la subtribu Bactridinae (Tomlinson, 1961).

El resto de los géneros, al presentar elementos 


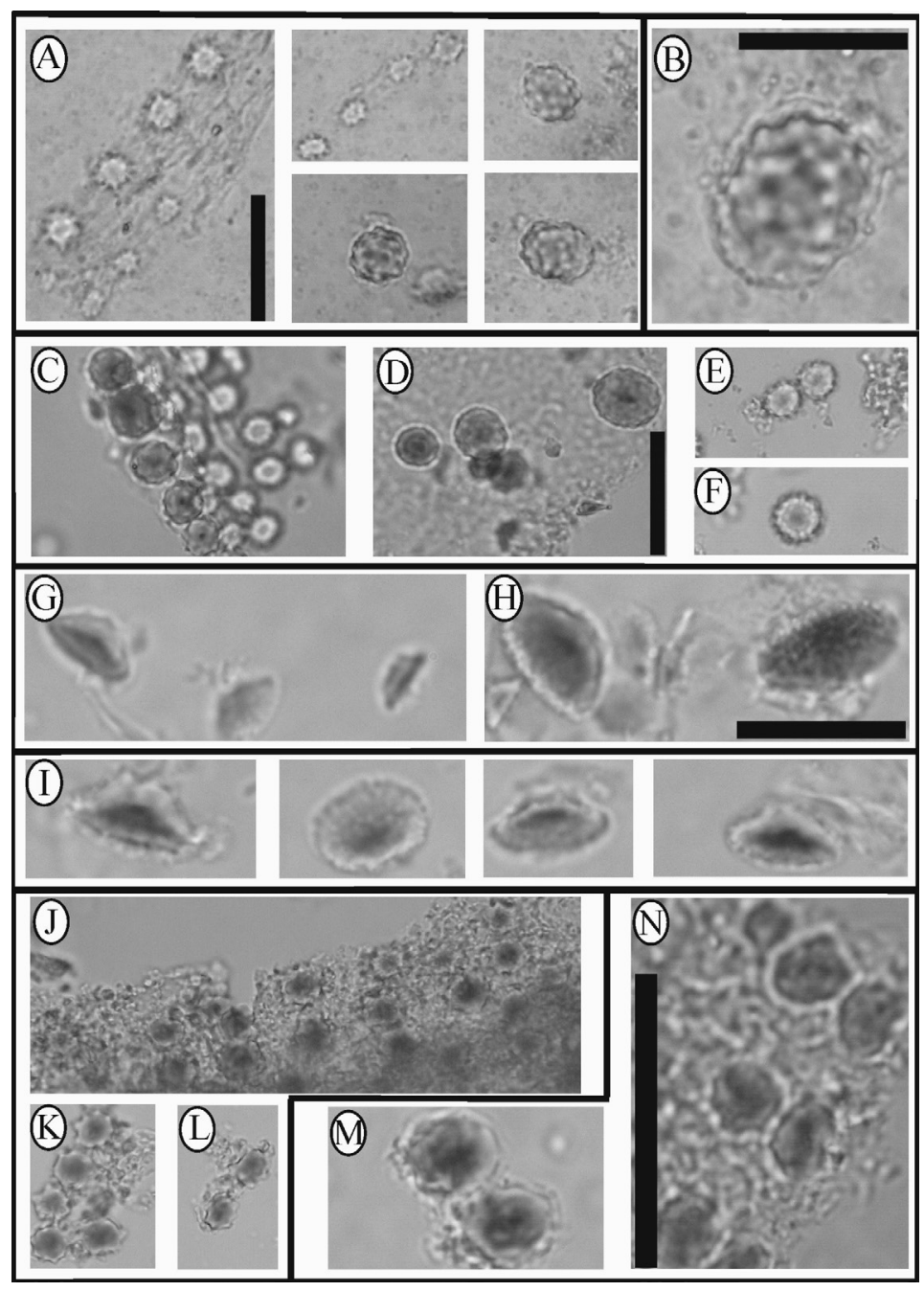

Fig. 4. A-B: Elementos globulares a elipsoidales de superficie espinosa (Cenizas de hoja de Euterpe edulis). C-F: Elementos globulares de superficie espinosa articulados y aislados (Cenizas de hoja de Copernicia alba). J-L: Elementos aislados y articulados de forma globular de bordes irregulares (Cenizas de hoja de Jubaea chilensis). G-I: Elementos de forma cónica (Cenizas de hoja de Acrocomia aculeata). Escala gráfica: A, B y D: $20 \mu \mathrm{m}$, en $\mathrm{H}$ y N: $10 \mu \mathrm{m}$.

globulares morfológicamente similares, resulta difícil diferenciarlos por características cualitativas sutiles, si bien mediante la tendencia general de los diámetros presentes en ellos pueden marcarse algunas variaciones de importancia. De esta manera las especies de la tribu Corypheae (T. campestris, $T$. brasilensis, T. schizophyla y C. alba) han presentado mayores tamaños de estos fitolitos con un valor promedio de 12,7 $\mu \mathrm{m}$. Por su parte, S. romanzzoffiana, B. yatay y B.capitata presentaron valores intermedios con promedios de $9,90 \mu \mathrm{m}$; mientras que E. edulis, A. campestris y J. chilensis presentaron valores promedios menores que rondaron los $7,4 \mu \mathrm{m}$.

Los análisis multivariados permitieron agrupar a las especies según sus asociaciones fitolíticas y las características biométricas medidas en cada una de ellas. El componente principal $1(67,98$ $\%$ de la variabilidad total) agrupa a las especies correspondientes con la subfamilia Arecoideae ( $B$. yatay, B. capitata, S. romanzzoffiana, E. edulis, 
Bol. Soc. Argent. Bot. 49 (4) 2014
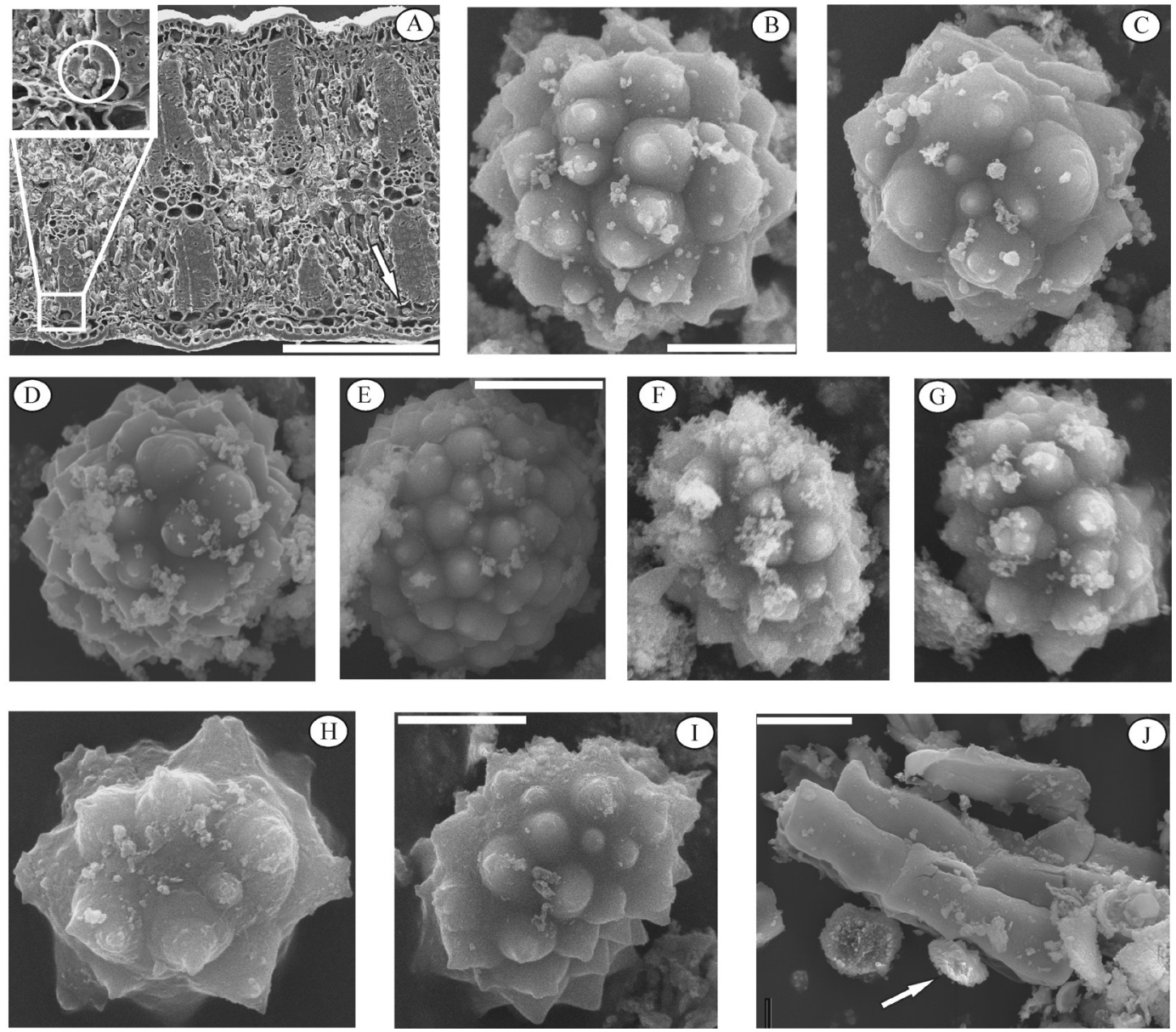

Fig. 5. Fotos de MEB. A: corte transversal de hoja de Butia yatay, la flecha indica la ubicación de los stegmatas en el tejido. Fitolitos globulares espinosos obtenidos a partir de cenizas de palmeras. B-C: Trithrinax campestris. D-E: Copernicia alba. F-G: Syagrus romanzzoffiana. H-I: Butia yatay. J: Elementos poliédricos y elemento cónico (indicado por la flecha) de Acrocomia aculeata. Escalas gráficas en A: 200 $\mu \mathrm{m} ; \mathrm{B}, \mathrm{E}, \mathrm{I}: 5 \mu \mathrm{m}$ y J: $1 \mu \mathrm{m}$.

J. chilensis y A. campestris), cuya variabilidad está dada por los fitolitos globulares cuyo rango de diámetros se encuentran entre los 5,08; 7,62 y $12,7 \mu \mathrm{m}$. Mientras que el componente $2(20,05$ $\%$ de la variabilidad total) agrupa a las especies de la subfamilia Corypheae (T. campestris, $T$. brasilensis, T. schizophylla y C. alba), donde la variabilidad está dada por los fitolitos globulares de $10,16 \mu \mathrm{m}$, aislando a $A$. aculeata del resto, dado al aporte principalmente de los fitolitos cónicos y las células epidérmicas. De esta manera se acentúa la relación entre las diferentes especies, tanto a nivel de subfamilia como de género (Fig. 7).

\section{Discusión}

Los resultados del presente trabajo, permitieron establecer a los morfotipos globulares espinosos como los principales integrantes de las asociaciones. Gran parte de éstos, tienen su superficie cubierta de cortas espinas y resulta difícil poder discriminarlos de una especie a otra al microscopio óptico.

El análisis biométrico permitió separar la tendencia de tamaños entre los diferentes órganos de un mismo ejemplar; de esta forma, la lámina foliar es el órgano que mayor cantidad de biomineralizaciones produce. En los análisis estadísticos, la media de 
N. E. Patterer - Análisis fitolíticos de palmeras
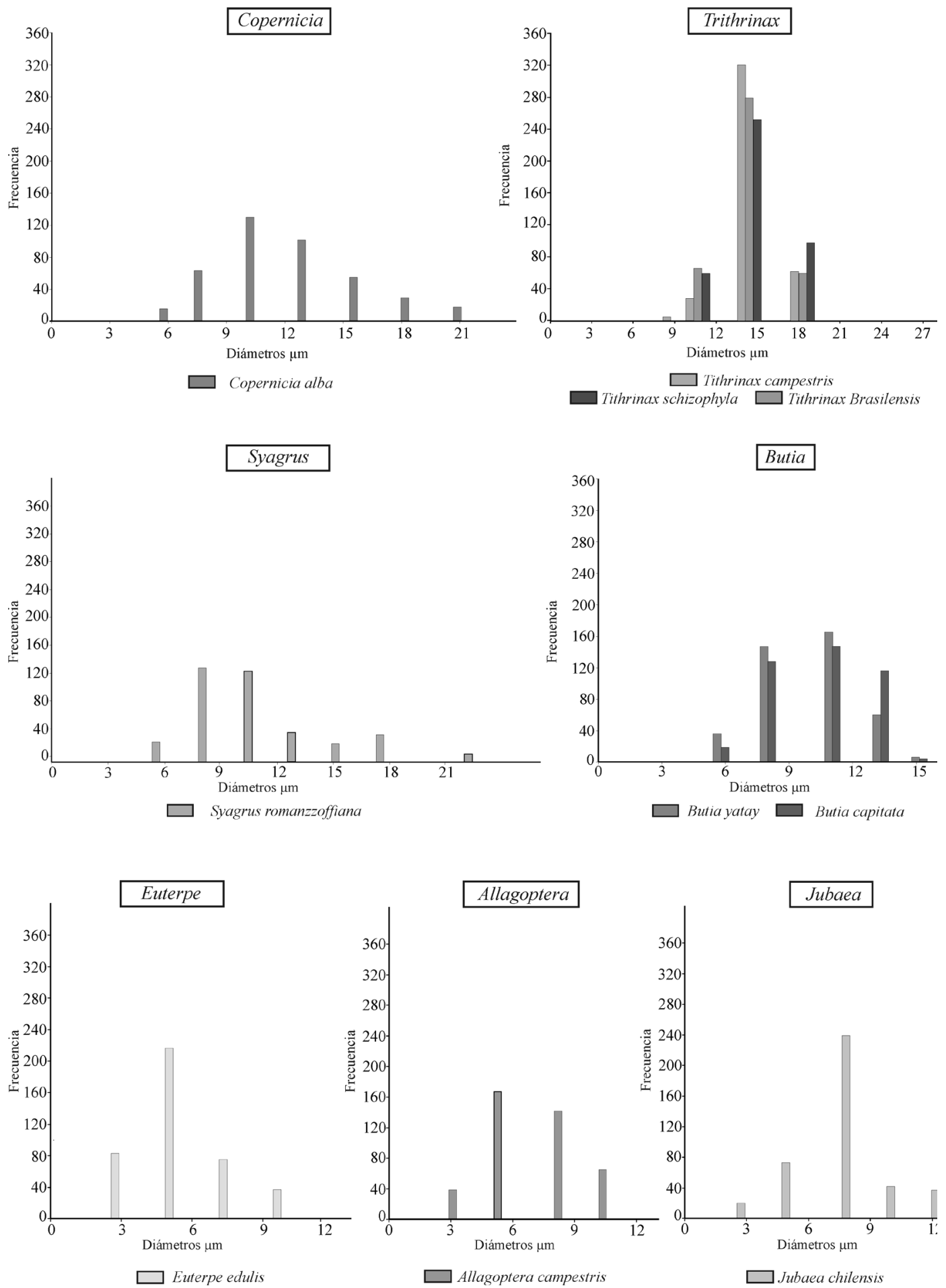

Fig. 6. Histogramas de frecuencias, mostrando las distribuciones de los diámetros de los fitolitos globulares en los distintos géneros. 
Bol. Soc. Argent. Bot. 49 (4) 2014

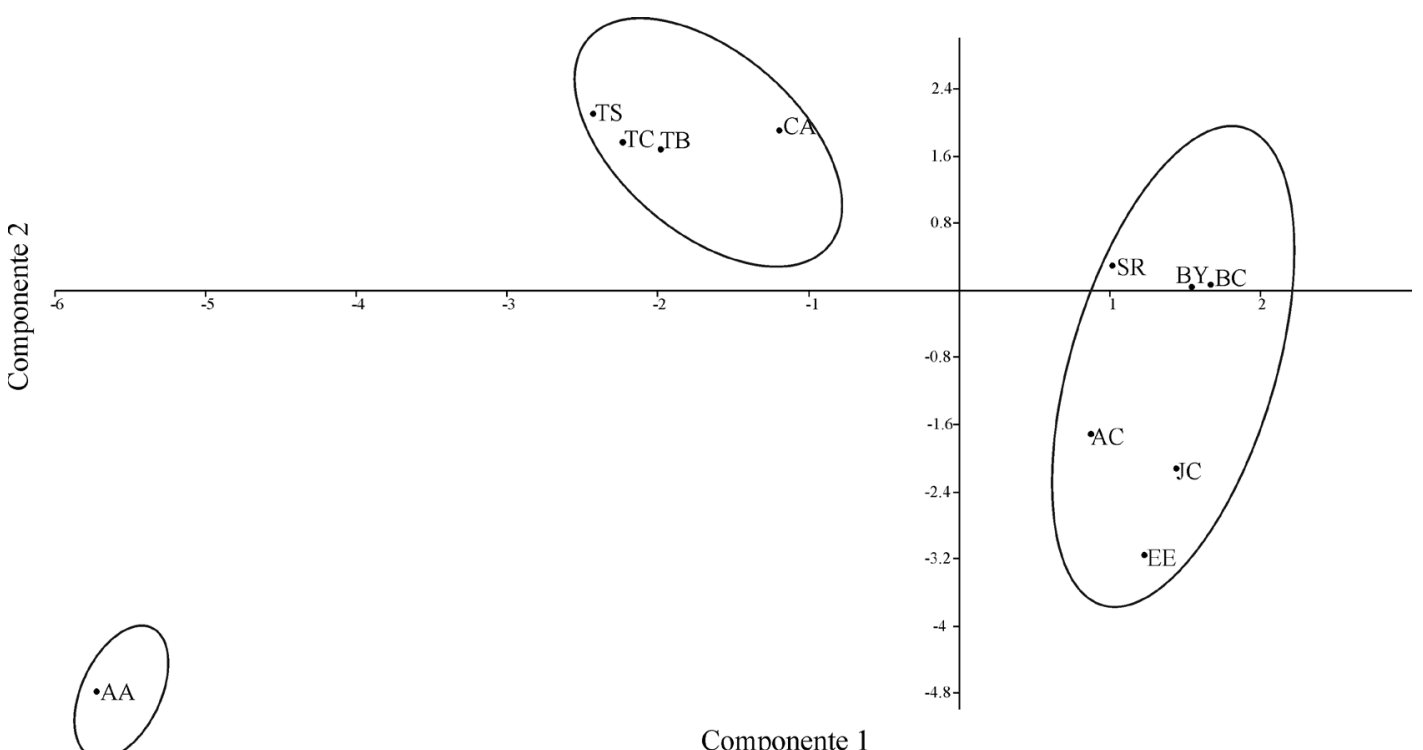

Fig. 7. Análisis de componentes principales de las diferentes especies de palmeras estudiadas. Trithrinax campestris (TC); T. brasilensis (TB); T. schizophylla (TS); Copernicia alba (CA); Butia yatay (BY); B. capitata (BC); Syagrus romazzoffiana (SR); Euterpe edulis (EE); Jubaea chilensis (JC); Allagoptera campestris (AC) y Acrocomia aculeata (AA).

Tabla 2. Descripción por especie de los fitolitos, rango de diámetros, descripción de las superficies y media estadística obtenida en el presente estudio. Porcentaje de presencia, A: Abundantes (50-100\%), R: Raros (10-50\%) y E: Escasos (<10\%).

\begin{tabular}{|c|c|c|c|c|c|c|c|}
\hline & & Fitol & itos & & & & \\
\hline Especie & Globulares & Elipsoidales & $\begin{array}{l}\text { Elementos de } \\
\text { conducción } \\
\text { y complejos } \\
\text { estomáticos }\end{array}$ & Cónicos & Superficie & Diámetros & Media \\
\hline Trithrinax campestris & A & $\mathrm{R}$ & $E$ & - & $\begin{array}{l}\text { Espinosa, de } \\
\text { ápices romos }\end{array}$ & 10 a $15 \mu \mathrm{m}$. & $12,5 \mu \mathrm{m}$ \\
\hline Trithrinax brasilensis & $A$ & $\mathrm{R}$ & $E$ & - & $\begin{array}{c}\text { Espinosa, de } \\
\text { ápices aguzados }\end{array}$ & 10 a $15 \mu \mathrm{m}$. & $12,6 \mu \mathrm{m}$ \\
\hline $\begin{array}{l}\text { Trithrinax } \\
\text { schizophylla }\end{array}$ & A & $\mathrm{R}$ & $E$ & - & $\begin{array}{l}\text { Espinosa, de ápices } \\
\text { redondeados }\end{array}$ & 10 a $15 \mu \mathrm{m}$. & $12,9 \mu \mathrm{m}$ \\
\hline Copernicia alba & A & $E$ & A & - & $\begin{array}{l}\text { Espinosa, de ápices } \\
\text { redondeados }\end{array}$ & 5 a $25 \mu \mathrm{m}$ & $12,5 \mu \mathrm{m}$ \\
\hline Euterpe edulis & A & $\mathrm{R}$ & $\mathrm{R}$ & - & Espinosa & 5 a $15 \mu \mathrm{m}$ & $5,5 \mu \mathrm{m}$ \\
\hline Butia yatay & A & E & A & - & $\begin{array}{l}\text { Espinosa, de } \\
\text { ápices agudos }\end{array}$ & 5 a $12 \mu \mathrm{m}$ & $9,5 \mu \mathrm{m}$ \\
\hline Butia capitata & A & $E$ & A & - & $\begin{array}{l}\text { Espinosa, de } \\
\text { ápices agudos }\end{array}$ & 5 a $12 \mu \mathrm{m}$ & $9,9 \mu \mathrm{m}$ \\
\hline Jubaea chilensis & A & A & $\mathrm{R}$ & - & Irregular & 2 a $10 \mu \mathrm{m}$ & $\begin{array}{c}6,5 \mu \mathrm{m} \\
10,5 \mu \mathrm{m}\end{array}$ \\
\hline $\begin{array}{l}\text { Syagrus } \\
\text { romanzzoffiana }\end{array}$ & A & A & A & - & $\begin{array}{l}\text { Espinosas, de ápices } \\
\text { redondeados }\end{array}$ & 5 a $25 \mu \mathrm{m}$ & \\
\hline $\begin{array}{l}\text { Allagoptera } \\
\text { campestris }\end{array}$ & A & $\mathrm{R}$ & $\mathrm{R}$ & - & Espinosas y lisas. & 2 a $12 \mu \mathrm{m}$ & $7,6 \mu \mathrm{m}$ \\
\hline Acrocomia aculeata & - & - & - & A & - & $10 \mu \mathrm{m}$ & - \\
\hline
\end{tabular}


los diámetros de los fitolitos globulares espinosos de la hoja, se corresponde con la media ponderada para el total de la planta, es decir, la hoja tendría el rango de medidas que se encuentran en el resto de los órganos estudiados. Autores como Bamford et al. (2006) observan diferencias en los fitolitos globulares presentes en diferentes partes de una misma planta, mientras que Delhon \& Orliac (2004) aplican métodos morfométricos cuantitativos para identificar fitolitos de raquis, hojas y troncos provenientes de diferentes palmeras nativas de la Isla de Pascua, al igual que en la presente contribución el análisis realizado a los diferentes órganos de $S$. romanzzoffiana, permitió encontrar diferencias entre los mismos.

El análisis biométrico demarcó una tendencia de tamaños en las diferentes especies analizadas, lo cual permitió agruparlas a nivel de subfamilia. Por ejemplo, en la subfamilia Arecoideae, Butia y Syagrus (subtribu Butiinae), presentan valores medios o tendencias de rango de diámetros entre los 9-10 $\mu \mathrm{m}$, mientras que Euterpe (subtribu Euterpinae), Allagoptera y Jubaea (subtribu Butiinae), están entre los 5-7 $\mu \mathrm{m}$; en el otro extremo de este rango, Tritrhinax y Copernicia (subfamilia Coryphoideae) son los géneros que presentaron los mayores diámetros con una media de $12 \mu \mathrm{m}$. Pearsall (2000) sugiere que no es posible identificar fitolitos de palmeras a nivel de género o de especies en áreas donde la diversidad de este tipo de la vegetación es extensas o ampliamente distribuidas (palmares). Para el presente estudio, el análisis permitió establecer una clara diferencia a nivel de subfamilia, caracterizando a las Coryphoideae con los valores medios más altos $(12 \mu \mathrm{m})$ y a la subfamilia Arecoideae, cuyas medias no superan los $10 \mu \mathrm{m}$. Fenwick et al. (2011), realizan un estudio piloto para la identificación de los morfotipos fitolíticos de palmeras y su aplicación a sitios arqueológicos. Toman especies económicamente importantes, donde dos de ellas pertenecen a la subfamilia Arecoideae (Areca catechu y Cocos nucifera), con valores medios de diámetros de fitolitos globulares entre 7 y $9 \mu \mathrm{m}$ y concluyen, que no es posible identificar un tipo morfológico a un nivel menor que no sea el de familia, proponiendo métodos estadísticos rigurosos a distintos caracteres morfológicos de los fitolitos foliares, que resultan dificultosos recabar mediante la observación en el microscopio óptico. No obstante, los primeros resultados obtenidos en este trabajo, en donde se han seleccionado especies de distintos integrantes de la familia, las medias de los diámetros de estos fitolitos han permitido diferenciar o agrupar las especies a nivel de subfamilia y en algunos casos de género. En síntesis, los valores medios encontrados para las diferentes especies, se corresponden con los valores encontrados por otros autores (Lins et al., 2002) para diferentes especies de estos géneros, por lo cual, responden más a una característica de la sistemática botánica de la familia, que a una razón de distribución o características del ambiente.

\section{Conclusión}

La producción de biomineralizaciones en el estudio de los diferentes órganos de un ejemplar, difiere en cantidad, siendo la hoja el mayor productor. Los diámetros de los fitolitos globulares en cada órgano estudiado fueron bastante homogéneos, mientras que la media ponderada del total de la planta se acerca más a la media registrada para las muestras de hoja.

El análisis biométrico comparativo entre las diferentes especies, permitió diferenciar y agrupar a las mismas a nivel de subfamilia y género, obteniendo así, una herramienta importante y apropiada para el estudio de este tipo de elementos en secuencias sedimentarias, sitios arqueológicos y/o unidades fisonómicas. Dado que los requerimientos ecológicos de los géneros estudiados son muy diferentes, esta contribución se presenta como un avance en este tipo de estudios, significativo desde el punto de vista de las reconstrucciones paleoambientales.

\section{Agradecimientos}

A los revisores M. Fernández Honaine y M. G. Fernández Pepi por sus valiosas contribuciones. A A. Zucol por su contribución en la realización de este trabajo. A M. Brea por la lectura crítica del manuscrito. A M. Arriaga y al herbario del Museo Argentino de Ciencias Naturales "Bernardino Rivadavia”, igualmente a C. Belmar, por facilitar material de Jubaea chilensis para el análisis presentado en este trabajo. El siguiente trabajo forma parte de la tesis doctoral de la autora, Facultad de Ciencias Físicas, Exactas y Naturales, UNC. Financiado por la Agencia Nacional de Promoción Científica y Tecnológica (Proyecto PICT $2008 \mathrm{~N}^{\circ}$ 0176). 


\section{Biblografía}

ALBERT, R. M., M. K. BAMFORD \& D. CABANES. 2009. Palaeoecological significance of palms at Olduvai Gorge, Tanzania, based on phytolith remains. Quatern. Int.193: 41-48.

BAMFORD, M. K., R. M. ALBERT, \& D. CABANES. 2006. Plio-Pleistocene macroplant fossil remains and phytoliths from Lowermost Bed II in the eastern palaeolake margin of Olduvai Gorge, Tanzania. Quatern. Int.148: 95-112.

DELHON, C. \& C. ORILAC. 2004. Extinct palms of Easter Island: the contribution of phytolith analysis. In: VI International Conference on Easter Island and the Pacific. Viña del Mar, Chile.

FENWICK, R. S. H., C. J. LENTFER, \& I. M. WEISLER. 2011. Palm reading: A pilot study to discriminate phytoliths of four Arecaceae (Palmae) taxa. J. Archaeo. Sci. 38: 2190-2199.

HAMMER, Ø., D. A. T. HARPER \& P. D. RYAN. 2001. PAST: Paleontological Statistics Software Package for Education and Data Analysis: Palaeont. Elect. 4: 9 pp.

LABOURIAU, L. G. 1983. Phytolith work in Brazil: a minireview. The Phytolit. News. 2: 6-10.

LINS, U., C. F. BARROS, M. DA CUNHA \& F. COSTA MIGUENS. 2002. Structure, morphology, and composition of silicon biocomposites inthe palm tree Syagrus coronata (Mart.) Becc. Protoplasma 220: 89-96.

MANCHESTER, S. R., LEHMAN, T. M. \& E. A. WHEELER. 2010. Fossil palms (Arecaceae, Coryphoideae) associated with juvenile herbivorous dinosaurs in the Upper Cretaceous Aguja formation, Big Bend National Park, Texas. Int. J. Plant Sci. 171, 679-689.

MARTÍNEZ, C. L. A. 2010. Estudios xilológicos en el Cretácico de la Cuenca Neuquina, Provincia de Neuquén, Argentina. Tesis para optar al Título de Doctor en Ciencias Naturales. Facultad de Ciencias Naturales y Museo. Universidad Nacional de La Plata.

MAZZONI, M. M. 1979. Contribución al conocimiento petrográfico de la Formación Sarmiento, barranca S del lago Colhué Huapí, provincia del Chubut. Rev. Asoc. Arg. Min., Petro. Sedim. 10: 33-53.

PATTERER, N. I., M. G. FERNÁNDEZ PEPI, A. F. ZUCOL \& M. O. ARRIAGA. 2009. Análisis de la Asociación Fitolítica De Pindó (Syagrus romanzzoffiana: Arecaceae). Bol. Soc. Argent. Bot. 44: 137.

PATTERER, N. I. 2012. Análisis Fitolíticos de la Formación El Palmar (Pleistoceno superior) en la Cuenca del Río Uruguay (este de Argentina). Tesis Doctoral. Facultad de Ciencias Exactas, Físicas y Naturales. Universidad Nacional de Córdoba.
PEARSALL, D. M., D. R. PIPERNO, E. H. DINAN, R. UMLAUF, Z. J. ZHAO \& R. A. BENFER. 1995. Distinguishing rice (Oryza sativa Poaceae) from wild Oryza species through phytolith analysis results of preliminary research. Econ. Bot. 49: 183-196.

PEARSALL, D. M. 2000. Paleoethnobotany. A handbook of procedures. $2^{\mathrm{a}}$. ed. Academic Press. New York,

PIPERNO, D. R. 1988. Phytolith Análisis. An Archaeological and Geological perspective. Academic Press. New York.

TOMLINSON, P. B. 1961. Anatomy of Monocotyldons. II: Palmae. Oxford, Clarendon Press. England.

UHL, N. \& J. DRANSFIELD. 1987. Genera palmarum. Allen Press. Kansas.

UHL, N. W. J. DRANSFIELD, J. I. DAVIS, M. A. LUCKOW, K. S. HANSEN \& J. J. DOYLE. 1995. Phylogenetic relationships among Palms: Cladistic analyses of morphological and chloroplast DNA restriction site variation. In: RUDALL, P. J., P. J. CRIB, D. F. CULTER \& C. J. HUMPHRIES (eds.). Monocotyledons: systematics and evolution. Royal Botanical Garden. Kew, U.K. 623-661.

ZUCOL, A. F., M. M. MAZZONI \& R. H. MADDEN. 1999. Análisis fitolíticos en la secuencia sedimentaria de Gran Barranca, Chubut. Ameghiniana 36: 43.

ZUCOL, A. F. \& M. BREA. 2000 a. Lignofloras del Cenozoico Superior del Noreste Argentino. In: ACEÑOLAZA G. \& R. HERBST (Eds.) El Neógeno de Argentina. Serie de Correlaciones Geológicas. 14: $245-253$.

ZUCOL, A. F. \& M. BREA. 2000 b. Análisis fitolítico de la Formación Paraná en Entre Ríos. In: ACEÑOLAZA, F. G. \& R. HERBST (eds.). El Neógeno de Argentina. Serie de Correlaciones Geológicas 14: 67 - 76.

ZUCOL, A. F., M. BREA, A. A.CARLINI \& R. H. MADDEN. 2001. Análisis fitolíticos en la secuencia sedimentaria de Gran Barranca, Chubut, Argentina: II. El Miembro Colhué Huapi (Formación Sarmiento). Ameghiniana 38 (4-suplemento): 50R.

ZUCOL,A. F., E. PASSEGGI, M. BREA, N. I. PATTERER, M. G. FERNÁNDEZ PEPI \& M. M. COLOBIG. 2010. Phytolith analysis for the patrok aike lake drilling proyect: sample treatment protocols for the pasado microfossil manual". In: H. CORBELLA \& N. I. MAIDANA (Eds.). $1^{\mathrm{a}}$ Reunión Internodos del Proyecto Interdisciplinario Patagonia Austral y 1er Workshop Argentino del Proyecto Potrok Aike Maar Lake Sediment Archive Drilling Project, Proyecto Editorial PIPA (Buenos Aires) 81-84.

Recibido el 7 de octubre de 2013, aceptado el 12 de noviembre de 2013 . 